\title{
CURRENT STATE OF ANTHROPOMETRIC PARAMETERS MEASUREMENT WITH RELEVANCE FOR ADVANCED LENS OPTOMETRIC COMPENSATION
}

\author{
George Baboianu ${ }^{1}$, Constantin Nitu ${ }^{2}$ and Constantin Daniel Comeaga ${ }^{3}$ \\ 1,2,3 University POLITEHNICA of Bucharest, Bucharest, Romania \\ email: geobaboianu73@gmail.com
}

\begin{abstract}
In recent years, in optometry, more emphasis is laid on the accurate measurements of the individual parameters depending on the main field activity of a patient during a working day. The lens built with individual parameters is a lens that gives the wearer maximum comfort. The devices for measuring these parameters have changed considerably, from an ordinary ruler for measuring the pupillary distance, to the latest generation devices that, by taking pictures, calculate all the needed parameters. The devices for parameters measuring are mobile or stationary units and include complex software that converts pixels that make up the picture took to the patient in distances. Hence, measurements can be entered into the software generator Free-Form of lens processing to customize them. The article presents the common problems in measurement of anthropometric parameters necessary for an advanced optometric compensation. The measurement methods, from very simple and cheap to actual image-processing based method, are presented with an emphasis on disadvantages and advantages of each one. Future direction of improvement is included.
\end{abstract}

Keywords: Optometric Compensation, Anthropometric Measurements, Free-Form Lens, ImageProcessing.

\section{Introduction}

\subsection{Optometric Parameters - Patient's Individual Parameters}

Ophthalmic optometry is the science that deals with the investigation of the functioning of the human visual system, analyzes the results, highlighting the problems, recommending methods and means of seeing improvement, in order to achieve visual comfort in relation to the needs of the subject, but without medical treatment, if possible [1]. Investigating the functioning of the visual system, analyses of the results, highlighting the problems, recommending the methods and means of improving the functioning, in order to achieve visual comfort in relation to the needs of the subject represents the science called Ophthalmic Optometry.

In recent years, optometry has developed more, considering the individual as an integral part of the living environment, analyzing the performance and visual problems daily, depending on the work developed during a day. The maximum visual performance achieved by a patient is due to the measuring methods and means of the optometric parameters. In recent years more emphasis is laid on the accurate measurements of the individual parameters depending on the main field activity of a patient during a working day. Since people have facial asymmetry, wearing a glasses frame is not exactly easy. There are people who have a deviated septum or differences in positioning of the ears and even a different horizontal and vertical positioning of the eyes. All these asymmetries that are commonly found in the patients' physiognomy must be taken into account in order to build a proper pair of glasses fit to the needs.

Parameters as inter-pupillary distance, vertex distance, convergence, are the parameters to be inserted into the lens calculation for a maximum comfort of the wearer. Also the chosen glasses frame and parameters are very important, now the new optical system consisting of the eyes and frame that holds the lens according to the prescription. The frame basic parameters are the pantoscopic angle and the curvature angle which, along with the constructive dimensions, represent an integrant part of the calculus formulas for a customized lens.

A precise optometric compensation based on modern free-form lenses, optimally designed and manufactured for each patient require an exactly evaluation of visual system and face dimensions. 


\subsection{Pupillary Distance}

The pupillary distance or the interpupillary distance is the distance measured in millimeters between the centers of the pupils and it is used in glasses prescription. Positioning the lenses correctly, according to the pupil center is vital to achieve optimal results in vision correction or for the improvement of the visual quality.

The pupillary distance value varies from one person to another taking into account gender, ethnicity, age, region and other values. There is a difference between men and women and between US and European or between Asia and America. Thus, for people in Europe, where Romania falls in, the normal value for adults is between 53 and $67 \mathrm{~mm}$ and for children between 40 and $54 \mathrm{~mm}$. These are the most common values which can be within normal limits, however the most common values among men in proportions of over $50 \%$ is of $69 \mathrm{~mm}$ and of $64 \mathrm{~mm}$ among women.[2]

Although the pupil is a circular hole located in the center of the iris diaphragm of anyone's eyes, its diameter normally varies between 2 to $5 \mathrm{~mm}$, according to the age (higher in children, lower in elders) and to the brightness to which the eye is exposed to, or in the case of certain functional states of the eye. It was observed that in $80 \%$ of people, the left and right intervals are not symmetrical, the difference reaching up to $5 \mathrm{~mm}$ (generally the left side is lower).

\subsection{Vertex BVD Distance (back vertex distance)}

Vertex distance, the distance between the cornea and the posterior surface of the lens, is the parameter necessary to simulate the movement of the eyes behind the lens and optimizing the visual areas. This vertex distance is of approximately 12 to $14 \mathrm{~mm}$, but it varies from person to person and there are many cases in which the same person has different vertex distances for each eye, separately.

The vertex distance is assumed in standard lens manufacturing of $12 \mathrm{~mm}$ and a positioning of the glasses frames closer or further away from your eyes, more or less influences, depending on the patient, the visual comfort(see Fig. 1.) [3].

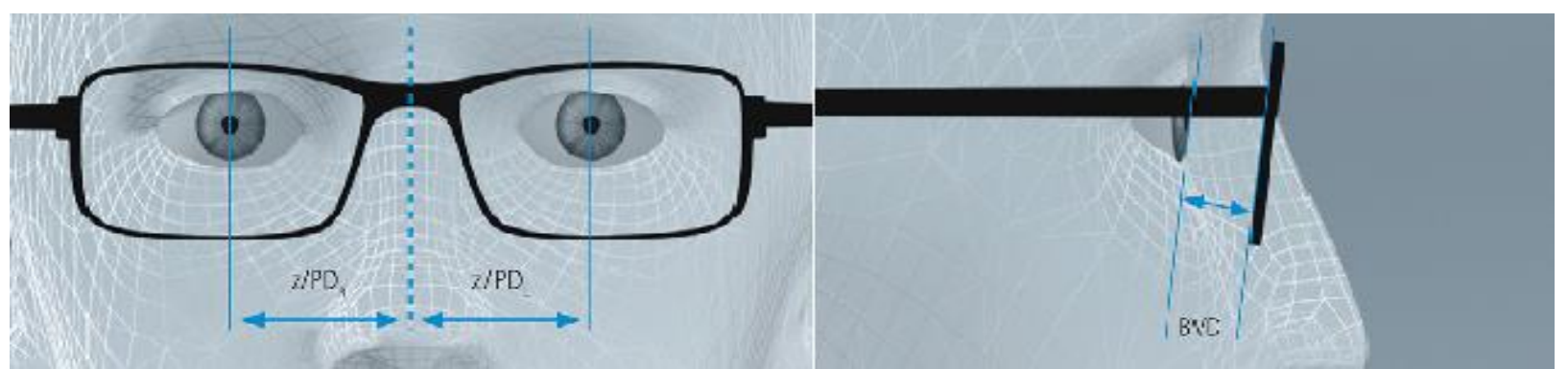

Figure 2: The pupillary distance on the center right and left interval, measured from the center of the nasal pyramid/Vertex distance - the distance between the plan tangent to cornea and the posterior tangential plan of the lens.[3]

\subsection{Convergence}

Convergence is described by the movement of the eyeball around its center of rotation. This movement of the eyeball occurs when the eye move the sight from infinity to close spaces. When we read or work within a small distance, convergence is maximum, pupils approaching the nose (see Fig. 2.). This movement of the eyeball is not identical in all individuals, convergence is more pronounced or not, depending on several factors such as the diameter of the eyeball, power accommodative various pathological conditions, etc.. There are people who don't have convergence. This rotation of the eyeball is of about $1.5 \mathrm{~mm}$. Some special devices measure convergence on each eye.

Convergence insufficiency is an imbalance in maintaining the binocular convergence. Paralysis of the convergence or the lack of convergence occurs after cranial-cerebral trauma, encephalitis, stroke, multiple sclerosis, diphtheria. It is characterized by double vision only for the close sight.

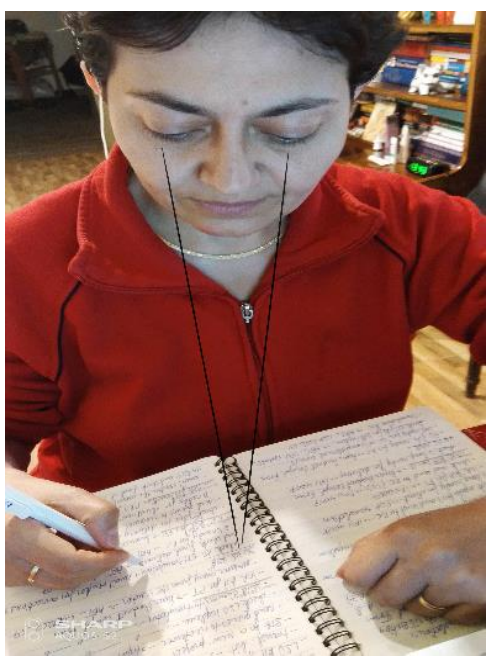

Figure 4: Convergence - looking to an object from a close distance. 


\section{Frame's Parameters}

The frame is very important and it is part of the visual system of the patient. The frame will be chosen according to physiognomy, to the material it is made of and depending on the lens that will be part of the assembly (see Fig. 3.). [3] The frame will be comfortable and must not slide off the face of the patient. For this there are taken into account the cheeks shape, the nose position, the eyebrows, and not least the eyes and ears position because there are fixed the support arms of the glasses frames.
In addition to the size and shape of the chosen frame, the parameters of the frame offer final details for a balance between the size and optical performance areas individualized visually and aesthetically. The variable height centering allows the selection of eyeglass frames of any size and allows the lens to be designed accordingly. A precise measurement of the inter-pupillary distance secures the visual area for the close sight.

In building of the customized progressive lenses there are taken into account all these design parameters and the following parameters for final positioning of the assembly.

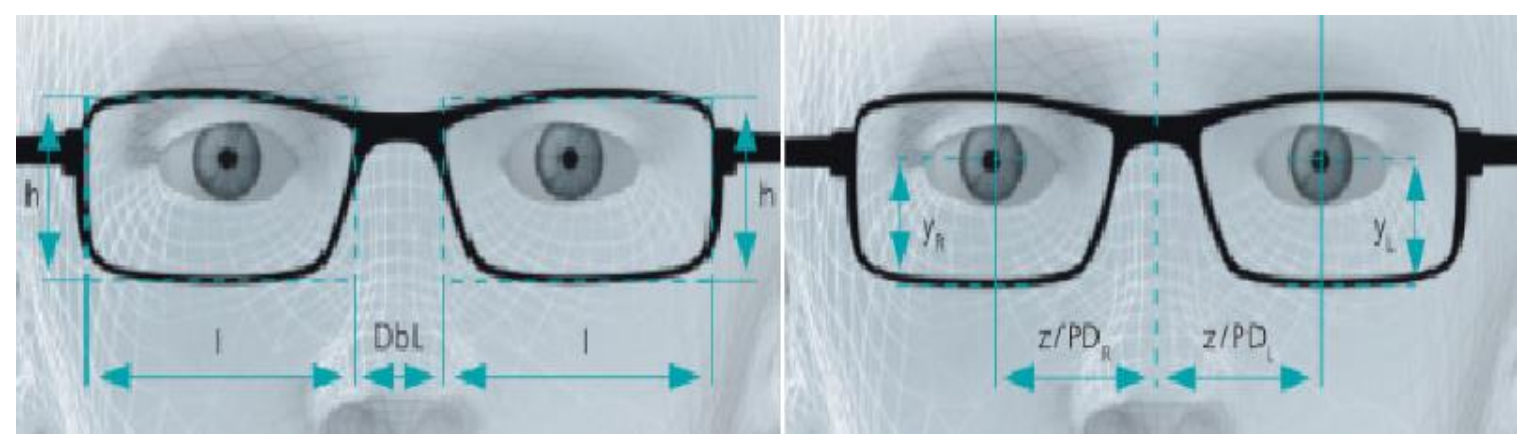

Figure 6: Constructive parameters of the glasses frame - front view [3].

\subsection{Curvature Angle of Frame}

This angle is described by the curvature radius of the two catches of the frame. For the glasses frames commonly used, this angle has little value between
0-15 degrees. The angle is greater for the frames used in sport or for protection, because it should cover a wider area and follow the shape of the face (see Fig. 4.).
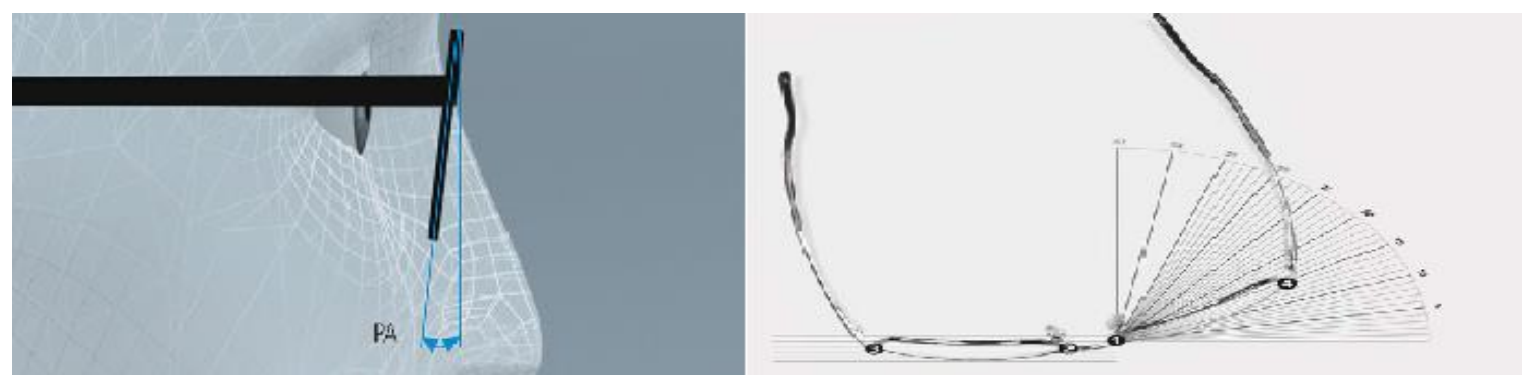

Figure 7: Thepantoscopic angle of the formed optical system / Curvature angle of the frame [3].

\subsection{Pantoscopic Angle}

The pantoscopic angle is the angle determined by the frame plane to the perpendicular plane to the optical axis of the eye looking to infinity; this angle has the standard value of 8 arc-degrees. This parameter may vary depending on the frame and on the physioanatomical parameters of the patient.

\section{Anthropometric Parameters}

According to the laws of nature, symmetry is a form of organization of inorganic and organic materials. But in the living beings and especially in the vertebrates, the body symmetry is not a constant.
In particular it was found that body shapes and especially human facial shapes are usually uneven this situation depends on the intrauterine position, vasculature and neurotroficity stimulation during the development period, hemispheric dominance that favors the skeleton and muscles of the dominant hemi-body.

\subsection{Standard facial parameters}

Although the slight facial asymmetry represent natural situations, they are usually hard perceptible. Even champions or world champions have small inequalities in terms of beauty of face shapes: uneven nostrils, small differences in the position of the ears, insert appendages, eye, etc.. 
The proportions between these characteristic dimensions, together with the color and facial figure, determine the individual physiognomy.

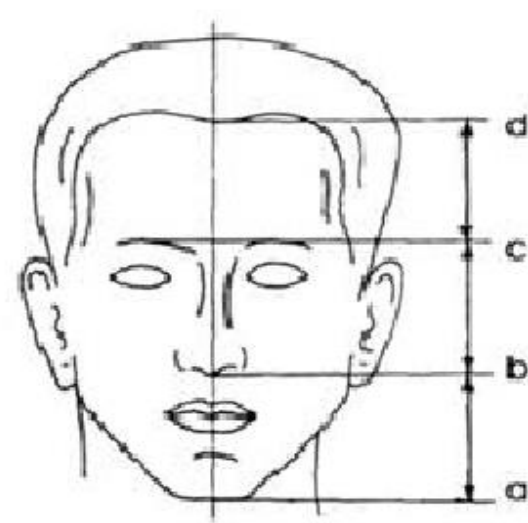

To accurately restore these proportions, there are used several anthropometric landmarks consisting in certain points, lines and planes located between the face and the figure limits (see Fig. 5.).

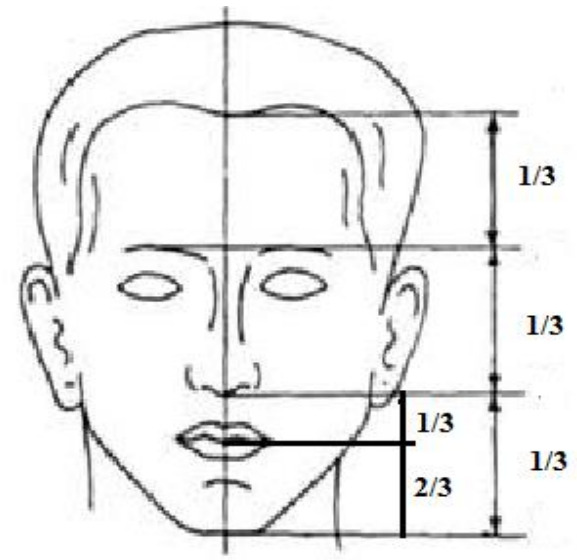

Figure 8: Delimitation of the face and figure / Standard aspect ratio of the face and figure.[3] $a-b-c=$ face; $a-b-c-d$ $=$ figure; $a-b=$ inferior side of the face and of the figure; $b-c=$ superior side of the face and middle side of the figure; $c-d=$ superior side of the figure.

\subsection{Facial asymmetry raises issues in} construction of glasses lenses

The facial asymmetries have neurological implications, genetic and neuro-vascular.

These syndromes are to be found both in adults and in childhood, puberty and adolescence. The neurologist knows the cranial nerve function and the autonomic nervous system, so he is the first who can determine the difference between anatomical and pathological variants. Anthropometric notes on facial abnormalities begin with standard measurements of the head and facial proportions, existing broad theories on normal and extreme faces to be framed in dysmorphia (see Fig. 6.).[4]

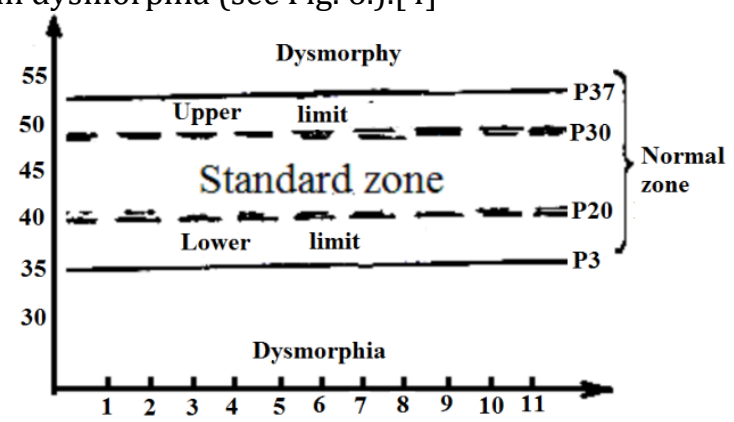

Figure 9: Scheme of the normal territory and facial dysmorphia according to the anthropometric measures. S. Stengel Rutkowski - 1985 [4].

In a classification commonly found in the medical literature, we find more than 100 craniofacial abnormalities marked by the morpho-anthropologic appearance, and among these there are described 25-28 with specific facial dysmorphia. Genetic facial abnormalities and embryonic malformations are clinically detected as facial cracks, atrophies or facial abnormalities or defects of the first pharyngeal mesoderm arch. Agenesis and dysgenesis of the branchial arches can affect the facial massif in whole or only on a half face. All these situations are facial asymmetries and defects. [4]

Septum deviations, the position in different planes of the eyeballs both vertically and horizontally, deformation of the cheeks, different positioning of the ears, are problematic in wearing a pair of glasses that leads to a special construction of the lens that takes into account all patient parameters.

\section{Measurement Techniques of Anthropometric and Final Spectacles Parameters}

In time, it was wanted that the anthropometric parameter measurements of the patient to be as exact as possible. This means a very precise construction of glasses and of course maximum comfort for the wearer. It started out as a distant interpupillary ruler, then the measurements were made mechanically and digitally.

A more precise ruler is the one that measures pupil position in relation to the vertical plane of nose symmetry in the area of the frame of the eyeglass.

The user place the special cutting on the middle of ruler on the nose base and measure from the zero point to the center of the left pupil and then to the center of the right pupil.

Such measurements are necessary due to multiple measurement errors with simple ruler from pupil reading errors due to low contrast between pupil and iris, eye movements, oblique 
reading and parallax error (removing it requires an eye to be covered by the patient and optometrist and open eye-to-open eye reading, which slows down the process and may cause problems for less wellmeaning patients, the elderly or the very young) to issues of poor communication between the patient and the optometrist, or to the fact that it is difficult to control the direction of the patient's eye and the distance to the target (see Fig. 7.).[5].
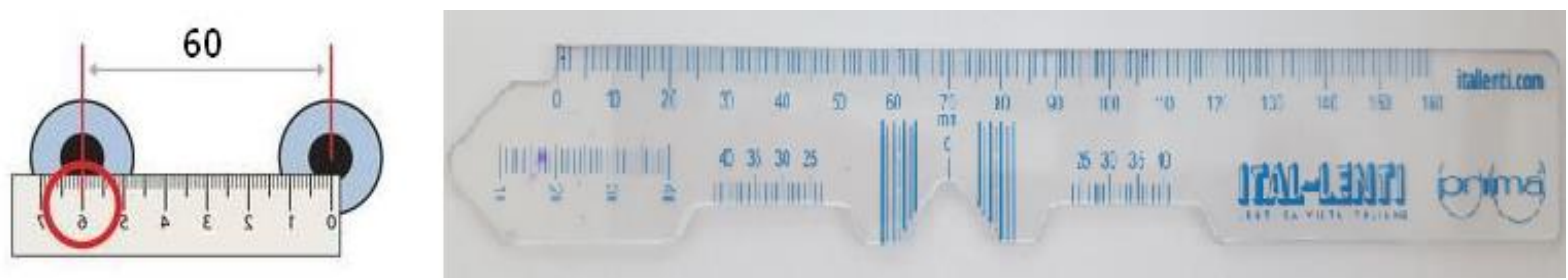

Figure 10: Measurement ruler for the pupillary distance / Special ruler for measuring pupil distance on left and right intervals [5].

With pupilometer is possible to solve the problem of distance to the target and the measurements are made for each eye, but it remains the problem of patient cooperation (it has fewer opportunities to distort the measurement but still exists). It is necessary to directly contact the device with the face of the patient but, most importantly, the measurements are made in a forced state by aligning the sight on a target in the device and having the right head), which may differ from its natural state of sight. At the same time, it is not possible to measure the positions in relation to the frame, which is very important both for the reduction of the errors in the calculation of the lenses but also because the conditions of the look change as the transition from the unobstructed look to the look in the compensating conditions and the glass eye.

Technology has advanced rapidly so precise devices have been built that can measure all the necessary parameters (see Fig. 8.) for personalized progressive lenses by taking photos.

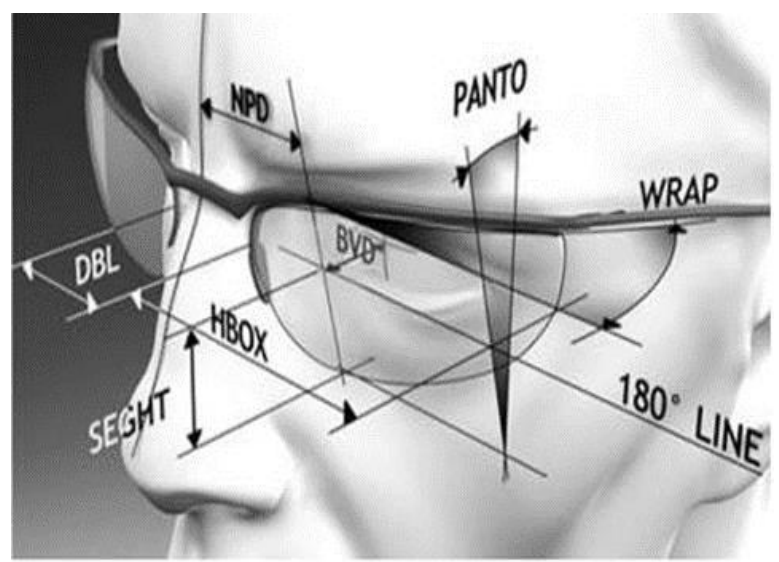

Figure 11: Individual parameters of the patient, inclusive of the selected frame [3].

All these individual parameters are used in the construction of the progressive lens in order to personalize it: interpupillary distance in the range, the vertex distance, pantoscopic angle, the convergence of each eye, radius of curvature of the frame, the height of installation, nasal bridge.

Measurements are made with a device that comprises specially designed software, processing images of patiente face with spectacles (see Fig. 9.).

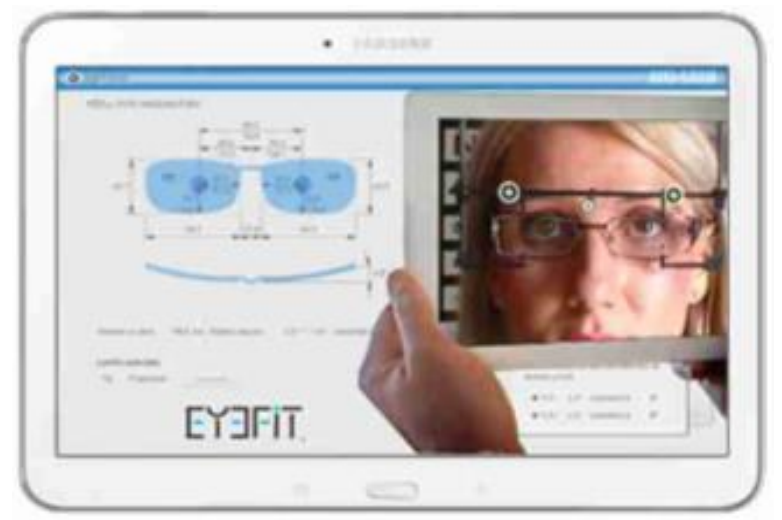

Figure 12: EYE FIT mobile device to determine the individual parameters [6].

Very important for making measurements is the frame clamp device, which has three markers positioned so that all measurements are calculated in relation to their layout.

The device is made of carbon and has 9 grams, making it easy not to change the position of the eyeglass frame it is catching. All dimensional and constructional and guiding angles of the device are known and will be part of the calculation of the measurements to be run by the software. It is very important to grip and position the device with the three guides, over the frame of the glasses chosen by the patient. Positioning is done taking into account that the marker in the center is exactly half the face and the selected frame. The device is required to build a correct position of the coordinate system in which the measurements are made and to establish image correction parameters. Without markers it is not possible to correctly determine the coefficients of the passage matrices from units in pixels to units in $\mathrm{mm}$ in space. 
Once the marker device has been correctly mounted on the eyeglass frame, the patient's pictures are taken(see Fig. 10.). The first picture is performed in front of the patient with his eyes in the tabletop room so that he can calculate the pupil distance to infinity for each interval. This also results in lens mounting height, eyeglass frame dimensions and nasal bridge size. There are two pictures in the profile, with the patient's eyes towards infinity, pictures that determine the frame pantoscopic angle, the frame curvature and the vertex distance for each eye. The last picture will be taken with the patient in close proximity to the tablet room to measure the following parameters: convergence on each eye and lens read position.

The software that runs on such a device is constructed to recognize numerous pixels forming the picture to turn the unit of measurement in $\mathrm{mm}$. The precision of measurements made with these devices is in terms of tenths of mm. After the patient was photographed, the EYE FIT program runs the received data and as a result we have all the patient measurements of the parameters and of the chosen frame parameters in order to build an ultrapersonalized progressive lens.
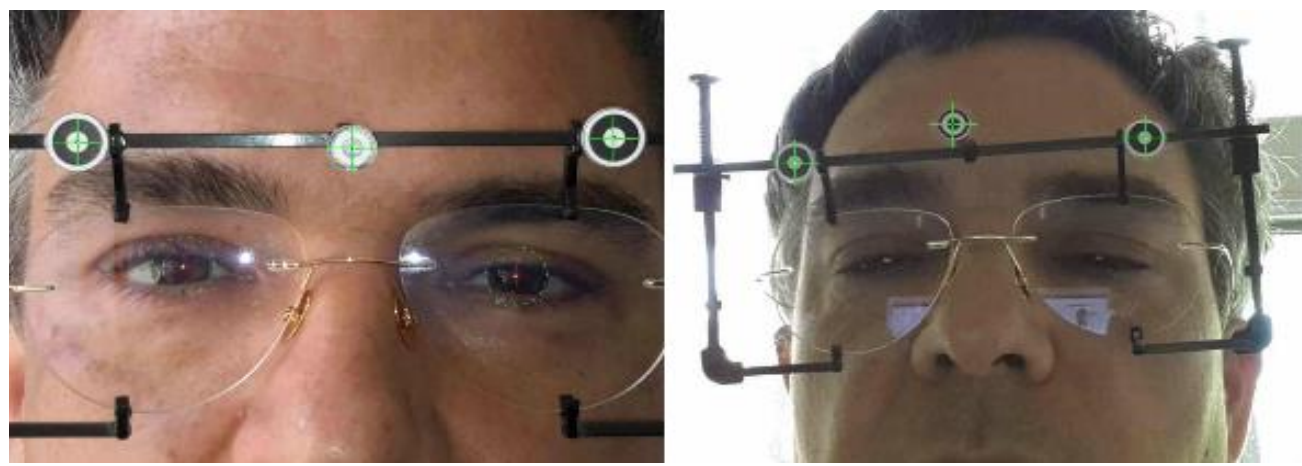

Figure 13: Example of images captured during measurement with EYE FYT - individual parameters - far sight and close sight measurements
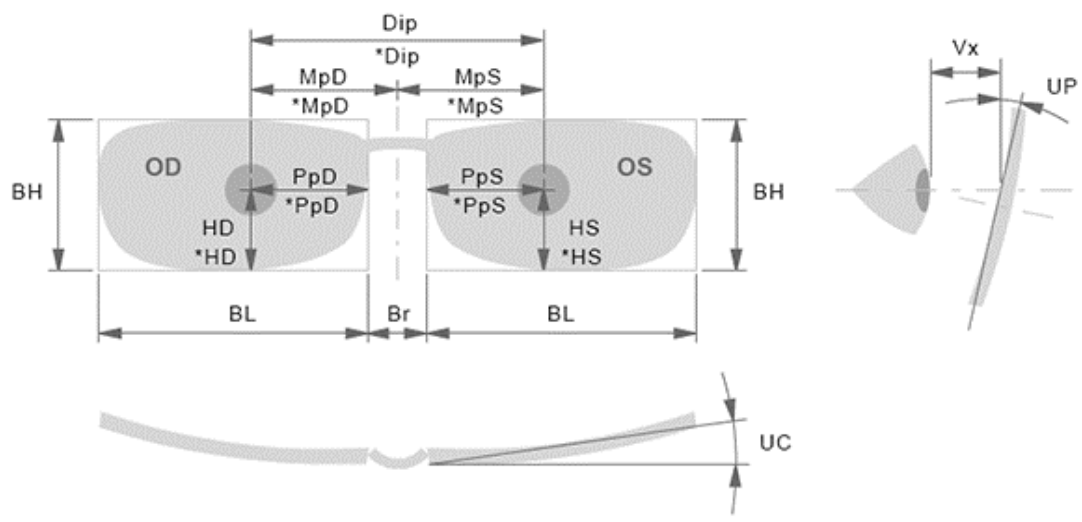

Figure 14: Example of close-sight individual parameters with EYEFYT after processing images

\begin{tabular}{|c|c|}
\hline Dip: & 70.7 \\
\hline MpD: & 34.9 \\
\hline MpS: & 35.8 \\
\hline PpD: & 25.7 \\
\hline PpS: & 26.8 \\
\hline HD: & 30.8 \\
\hline HS: & 28.4 \\
\hline
\end{tabular}

\begin{tabular}{|c|c|}
\hline $\mathrm{BH}$ : & 42.5 \\
\hline BL: & 55.1 \\
\hline $\mathrm{Br}$ : & 17.7 \\
\hline$V x$ & 22.15 \\
\hline VxOD: & 22.7 \\
\hline VxOS: & 21.6 \\
\hline UP: & 5.4 \\
\hline UC: & 4.6 \\
\hline
\end{tabular}

* Close sight distancees

*Dip: $\quad{ }^{*} 68.3 \mathrm{~mm}$

*MpD: $\quad{ }^{*} 33.9 \mathrm{~mm}$

"MpS: $\quad{ }^{*} 34.4 \mathrm{~mm}$

*PpD: $\quad{ }^{*} 23.1 \quad \mathrm{~mm}$

*PpS: $\quad{ }^{*} 23.5 \mathrm{~mm}$

*HD: $\quad{ }^{*} 24.2 \mathrm{~mm}$

*HS: $\quad{ }^{*} 22.4 \mathrm{~mm}$

Reading distance : * $36.5 \mathrm{~cm}$

Figure 15: Example of measurements of the individual parameters measured by EYE FIT. Reading distance; Dip =interpupillary distance; $M p D=$ interpupillary distance for the right eye; $M p S=$ interpupillary distance for the left eye; $V x=$ vertex distance (from the cornea to the lens); $H D, H S=$ The distance from the pupil to the tangent in the

lens; $B H=$ Height of the frame; $B L=$ Frame length; $B r=$ bridge; UP = pantoscopic angle; UC =frame angle of curvature 
Differences of parameter measurements are observed between the two halves of the face. For people with large differences in parameters, special lenses are built to give them comfort and visual field to meet their work needs.

\section{Conclusions}

Technology is constantly evolving. The help that man receives from advanced technological equipment is substantial. In the field of ophthalmology, technology helps us in accurate determinations of pathologies not detected with the naked eye. Technological evolution shows that new knowledge and technological advances in image acquisition, computing power in portable devices, the explosive development of mathematical fundamentals and image processing algorithms, and non-contact spatial measurements have allowed the passage of to manual measurements affected by numerous errors, to automatic, performance measurements.

However, it should be noted that there are still major issues related to patient collaboration, uneven illumination, the need to achieve several successive pictures with patient resting without any movement and their processing.

Consequently, advanced studies are needed to improve the method, to reduce the number of shots (by improving algorithms to be more robust in relation to error sources and to making an optical system with multiple micro-cameras permitting the simultaneous execution of more photos).

\section{Acknowledgement}

The authors thank Nova Lenti company and, in particular, Mr. Mihai Leu for permanent support during this study.

\section{References}

[1] Dumitrescu N., Bazele opticii fiziologice, Ed. Politehnica, Bucureşti (1991).

[2] Bioptical art Homepage, http://www.artabioptica.ro/art3.htm, last accessed 2018/05/21

[3] Technical-commercial documentation/catalogue - Carl Zeiss

[4] Stengel-Rutkowski S., Schimanek P., Chromosomal und nicht-chromosomale Dysmorphiesyndrome, EnkeVerlag, Stuttgardt, (1985).

[5] Ophtalmi'x Blog Homepage, https://oftalmix.wordpress.com, last accessed 2018/05/21

[6] Soft EYE FIT - C CENTWINS SRL, www.optivio.ro, last accessed 2018/05/21. 